\title{
QUANTIFICATION OF TIDAL WATERTABLE OVERHEIGHT DUE TO THE SLOPING BEACH IN UNCONFINED AQUIFERS
}

\author{
Zhiyao Song ${ }^{1}$ Zhuo Zhang² and Ling $\mathrm{Li}^{3}$
}

\begin{abstract}
Based on a liberalized one-dimensional Boussinesq model and the previous study results, this paper provides two experience solutions to quantify the tidal groundwater overheight behind arbitrary sloping beaches. One solution is obtained with an asymptotic matching method advanced by Guo, and the other solution is origined by the analytical solution as perturbation parameter less than unity, the errors of both solutions compared with numerical solution are small and acceptive for the application.
\end{abstract}

Keywords: Coastal aquifer; Tidal watertable; Boussinesq equation; logarithmic matching; experience solution

\section{INTRODUCTION}

Generally, tidal watertable overheight or super-elevation is defined as the difference between the time-averaged watertable and the mean sea level (MSL) for far inland in the absence of regional groundwater flow. As one of the principal features of the groundwater response to tidal forcing in a coastal unconfined aquifer, which is found both in field observations and indoor experiments and demonstrated through theoretical researches, it is an important parameter related to a thorough understanding of the interaction between tides and the groundwater flow, such as the formation of groundwater circulation, the intrusion of saltwater, the erosion or aggregation of beachface, the estimation of submarine groundwater discharge (SGD) and associated chemical input to the coastal ocean, specially for the very flat beaches (e.g., Phillip, 1973; Parlange et al., 1984; Nielsen, 1990; Church, 1996; Moor, 1996; Li et al., 2000; Song et al., 2006; Roberts,2008).

The generation of tidal watertable overheight is attributed to the formation of seepage face on the beachface, a moving boundary condition due to the beach slope and the non-linearity of tide-induced groundwater wave propagation (e.g., Phillip, 1973; Parlange et al., 1984; Nielsen, 1990; Li et al., 2000; Song et al., 2006). Because the seepage face formation is not well undersood and the nonlinear effects on tidal propagation in the aquifer with the vertical beach is demonstrated by perturbation solutions (e.g., Parlange et al., 1984; Song et al., 2007), we focus on the quantification of the tidal watertable overheight due to the sloping beach in this paper.

Based on a linearised one-dimensional Boussinesq model and the previous study results, this paper provides two experiense solutions to quantify the tidal groundwater overheight behind arbitrary sloping beaches.

\section{BRIEF REVIEW OF PREVIOUS STUDIES}

We consider one-dimensional shallow groundwater flow in an unconfined coastal aquifer with a horizontal impermeable base to be homogeneous, isothermal and incompressible, and consider only small amplitude tides compared with the aquifer thickness, which is a valid assumption in most cases. This flow is often modelled by a linearised Boussinesq equation (e.g., Bear, 1972; Nielsen, 1990; Li et al., 2000; Song et al. 2006)

$$
\frac{\partial h}{\partial t}=\frac{K D}{n_{e}} \frac{\partial^{2} h}{\partial x^{2}}
$$

where $x[\mathrm{~L}]$ is the horizontal inland coordinate normal to coastline; $t[\mathrm{~T}]$ is time; $h(x, t)[\mathrm{L}]$ is the watertable height from the MSL (Fig.1) ; $D$ is the mean aquifer thickness, $K$ is the saturated hydraulic conductivity, and $n_{e}$ is the effective porosity (all assumed to be constants).

\footnotetext{
${ }^{1}$ Key Laboratory of Virtual Geographic Environment (Ministry of Education) Nanjing Normal University,1 Wenyuan Road, Xianlin, Nanjing, 210097, China.

${ }^{2}$ College of Harbor, Coastal and Offshore Engineering, Hohai University, 1 Xikang Road, Nanjing, 210098, China.

${ }^{3}$ School of Engineering, The University of Queensland, St.Lucia, Brisbane, OLD4072, Australia
} 


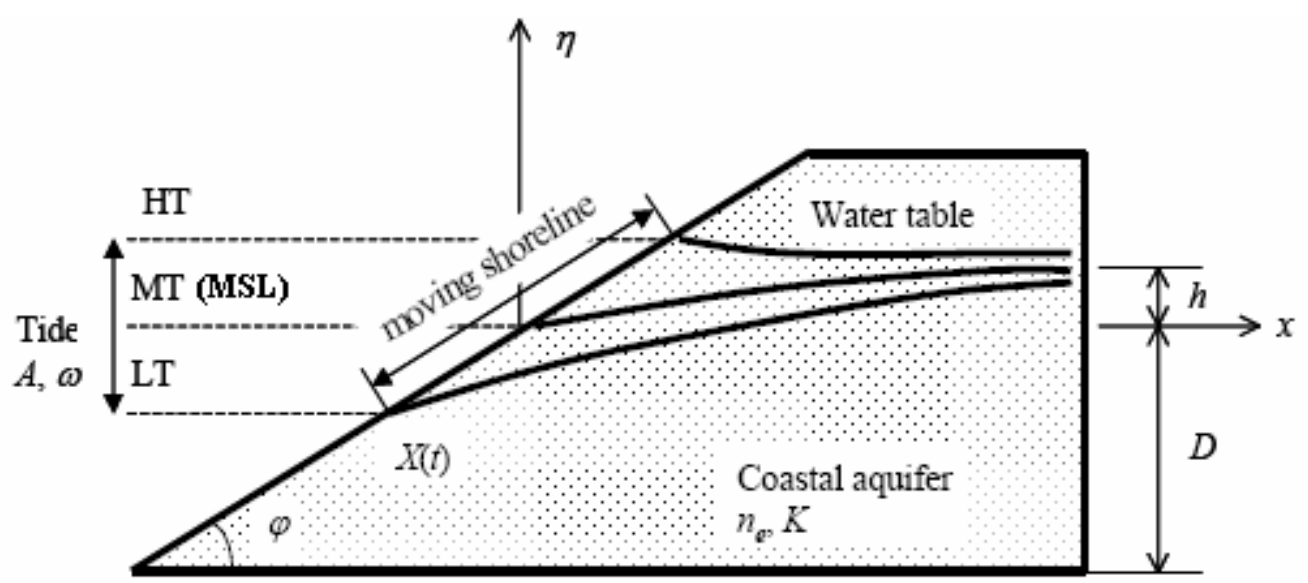

Figure 1. Schematic diagram of tidal watertable fluctuations in a coastal aquifer (HT, MT and LT denote the high, mid and low tidal level )

If the seepage face and regional groundwater head gradient (flow) are negligible, the moving boundary condition is given by

$$
h\left[x_{0}(t), t\right]=\eta(t)=A \cos (\omega t) \text { and } x_{0}(t)=\cot (\varphi) \eta(t)
$$

where $x_{0}(t)$ is the $\mathrm{x}$-coordinate of the moving boundary; $\varphi$ is the slope of the beach; $\eta(t)$ represents tidal oscillations of the MSL; A is the tidal amplitude; and $\omega$ is the tidal frequency.

By introducing the new variable $Z=x-x_{0}(t)$, Eqs. (1) and (2) can, respectively, be transformed to (Li et al., 2000)

$$
\begin{aligned}
& \frac{\partial h}{\partial t}=\frac{K D}{n_{e}} \frac{\partial^{2} h}{\partial z^{2}}-A \omega \cot (\varphi) \sin (\omega t) \frac{\partial h}{\partial z} \\
& h(0, t)=A \cos (\omega t)
\end{aligned}
$$

Thus, the moving boundary problem of Eq. (1) is mapped to a fixed boundary problem.

Far inland ( $z \rightarrow \infty$ ), we consider only the tidal effects, the gradient of $h$ is taken to be zero (the tidal effects are diminished), i.e.,

$$
\left.\frac{\partial h}{\partial z}\right|_{z \rightarrow \infty}=0
$$

For the purpose of simplicity and generality, the following non-dimensional variables are introduced:

$$
x^{*}=z / L, h^{*}=h / A \text { and } t^{*}=\omega t
$$

where $L=\sqrt{\frac{K D}{n_{e} \omega}}$ represents a decay length scale of watertable fluctuations.

Substituting (6) to (3) gives

$$
\frac{\partial h^{*}}{\partial t^{*}}=\frac{\partial^{2} h^{*}}{\partial x^{* 2}}-\sqrt{2} \varepsilon \sin \left(t^{*}\right) \frac{\partial h^{*}}{\partial x^{*}}
$$

and the corresponding boundary conditions as follows

$$
\begin{aligned}
& h^{*}\left(0, t^{*}\right)=\cos \left(t^{*}\right) \\
& \left.\frac{\partial h^{*}}{\partial x^{*}}\right|_{x^{*} \rightarrow \infty}=0
\end{aligned}
$$


where $\varepsilon=A \cot (\varphi) /(\sqrt{2} L)=A \cot (\varphi) \sqrt{\frac{n_{e} \omega}{2 K D}}$ is a dimensionless parameter, combining all participating dimensional parameters, which is used by Nielsen (1990) and Teo et al. (2003) in their analytical solution investigation.

For $\varepsilon<1$, using the perturbation method with Li et al.(2000) the sixth order dimensionless overheight solution $\overline{h_{\infty}^{*}}(\varepsilon)$ can be determined

$$
\overline{h_{\infty}^{*}}(\varepsilon)=\frac{1}{2} \varepsilon-\frac{\sqrt{2}-1}{4} \varepsilon^{3}+\frac{12 \sqrt{6}-18 \sqrt{3}+28 \sqrt{2}-31}{192} \varepsilon^{5}+O\left(\varepsilon^{7}\right)
$$

For $\varepsilon<3$, Song et al. (2006) presents a new analytical approach to solving above Boussinesq equation with the Fourier-series expansion, and values of the dimensionless overheight are given.

For $\varepsilon \rightarrow \infty$, employing a modified Crank-Nicholson predictor-corrector method for the advection-diffusion equation (Ames, 1977; Parlange et al. 1984), the numerical solution of (7) with the boundary condition (8) and (9) can be obtained easily. The results show the dimensionless overheight equals the unit as $\varepsilon$ approaches infinity. Based on the Fourier-series expansion, this numerical result is proved further by Song et al.(2006) in theory.

As abovemention, previous studies of moving-boundary effects on watertable overheight don't give a mathematical function for $0 \leq \varepsilon \leq \infty$, so these results is hard to be applied in the practice.

\section{EXPERIENCE SOLUTION}

From the results of previous studies, we can find two asymptotes as follows

(1)Analyzing the solution (10), the dimensionless overheight solution $\bar{h}_{\infty}(\varepsilon)$ can be expanded as an alternative series about $\varepsilon$, and $\overline{h_{\infty}^{*}}(\varepsilon)=\frac{1}{2} \varepsilon$ as $\varepsilon \rightarrow 0$.

(2)Through numerical solution and mathematical proof, the expanded series of $\bar{h}_{\infty}(\varepsilon)$ should be converged, and $\overline{h_{\infty}^{*}}(\varepsilon) \rightarrow 1$ as $\varepsilon \rightarrow \infty$.

Using the logarithmic matching, an asymptotic matching method advanced by Guo (2003), one can show that the dimensionless overheight solution $\overline{h_{\infty}^{*}}(\varepsilon)$ is

$$
\overline{h_{\infty}^{*}}(\varepsilon)=\frac{\varepsilon}{2}\left[1+\left(\frac{\varepsilon}{\varepsilon_{0}}\right)^{\beta}\right]^{-1 / \beta}
$$

in which $\varepsilon_{0}=2$ is determined by the asymptote (2), and $\beta=5 / 4$ is solved from (11) at $\varepsilon=10$ where the corresponding numerical value is $\overline{h_{\infty}^{*}}(10)=0.90$.

The other solution is pure experience. Assuming $\overline{h_{\infty}^{*}}(\varepsilon)$ as

$$
\overline{h_{\infty}^{*}}(\varepsilon)=a \tan ^{-1}(b \varepsilon)
$$

we have $a b=\frac{1}{2}$ with asymptote (1) and $\frac{\pi}{2} a=1$ with asymptote (2), that is, $a=\frac{2}{\pi}$ and $b=\frac{\pi}{4}$.

Expanding solution (12) into a power series of $\varepsilon$ within [0,1], we find surprising its coefficients are very close to those of solution (10) up to the sixth order.

The experience solutions (11) and (12) are compared in Fig.2 with numerical solution. This comparison show the solution (11) is better slightly than the solution (12) fitted to numerical solution, but both errors are acceptive for the application. 


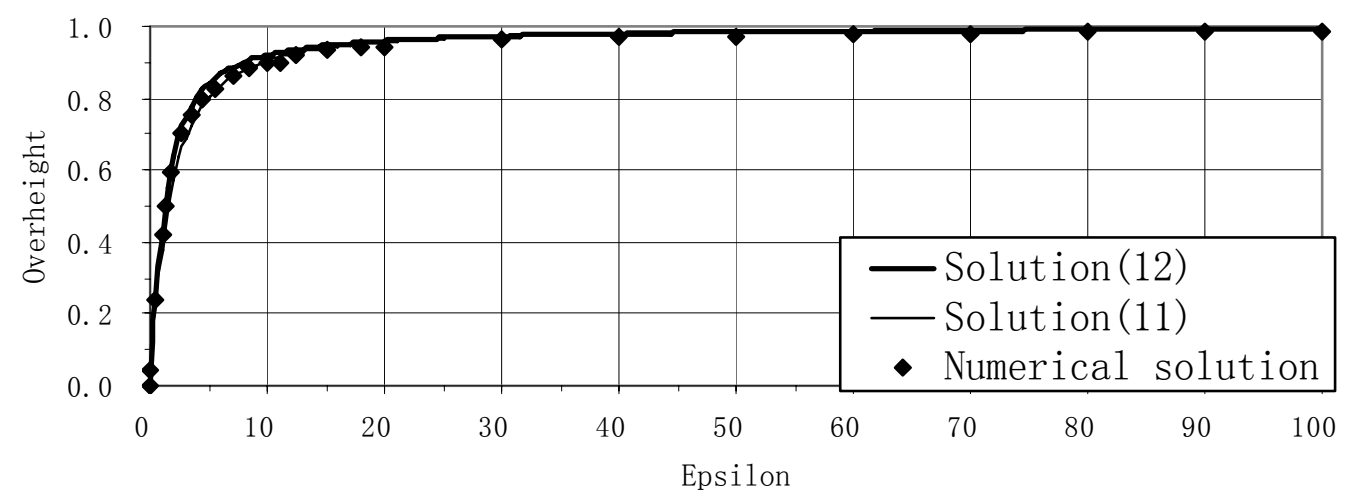

Fig.2 Comparison of experience solutions and numerical solution

\section{Conclusion}

In order to determine the inland overheight $\bar{h}_{\aleph}(\varepsilon)$, we have given two experience solutions (11) and (12) by numerical computing for $0 \leq \varepsilon<100$ using (7) with the corresponding condition (8) and (9). One is obtained with an asymptotic matching method, the other is origined by the analytical solution (10) for $0 \leq \varepsilon<1$, the errors of both solutions are small and acceptive for the practice.

\section{ACKNOWLEDGEMENT}

This study is supported by the Natural Science Foundation of the Jiangsu Higher Education Institutions of China(Grant No.09KJA170003 ).

\section{REFERENCES}

Church, T. M., 1996, A groundwater route for the water cycle. Nature, 380, 579-580.

Guo, J., 2002, Logarithmic matching and its applications in computational hydraulics and sediment transport. J. Hydr. Res., 40(5), 555-565.

Li, L., D. A. Barry, F. Stagnitti, J.-Y. Parlange, and D.-S. Jeng, 2000, Beach water table fluctuations due to spring-neap tides: moving boundary effects. Adv Water Resour, 23, 817-824.

Moore, W. S., 1996, Large groundwater inputs to coastal waters revealed by 226Ra enrichment. Nature, 380, 612-614.

Nielsen, P., 1990, Tidal dynamics of the water table in beaches. Water Resour. Res., 26, 2127-2134.

Parlange, J.-Y., F. Stagnitti, J. L. Starr and R. D. Braddock, 1984, Free-surface flow in porous media and periodic solution of the shallow-flow approximation. J. Hydrol., 70, 251-263.

Philip, J. R., 1973, Periodic nonlinear diffusion: An integral relation and its physical consequences. Aust. J. Phys., 26, 513-519.

Roberts, M. E., 2008, Groundwater response to tidal forcing. ANZIAM J. 50(CATA2008), C640C653.

Song, Z. Y., L. Li, P. Nielsen and D. Lockington, 2006, Quantification of tidal watertable overheight in an unconfined coastal aquifer. J. Eng. Math., 56, 437-444.

Song, Z. Y., L. Li, J. Kong and H. G. Zhang, 2007, A new analytical solution of tidal water table fluctuations in a coastal unconfined aquifer. J. Hydrol., 340, 256-260.

Teo, H. T., D. S. Jeng, B. R. Seymour, D. A. Barry and L. Li, 2003, A new analytical solution for water table fluctuations in coastal aquifers with sloping beaches. Adv Water Resour, 26, 12391247. 